\title{
Article
}

\section{Low-energy electron diffraction study of potassium adsorbed on single-crystal graphite and highly oriented pyrolytic graphite}

Ferralis, N., Pussi, K., Finberg, S., Smerdon, Joe, Lindroos, M., McGrath, R. and Diehl, R.

Available at https://clok.uclan.ac.uk/9499/

Ferralis, N., Pussi, K., Finberg, S., Smerdon, Joe orcid iconORCID: 0000-00027387-8362, Lindroos, M., McGrath, R. and Diehl, R. (2004) Low-energy electron diffraction study of potassium adsorbed on single-crystal graphite and highly oriented pyrolytic graphite. Physical Review B, 70 (24). ISSN 10980121

It is advisable to refer to the publisher's version if you intend to cite from the work. http://dx.doi.org/10.1103/PhysRevB.70.245407

For more information about UCLan's research in this area go to http://www.uclan.ac.uk/researchgroups/ and search for <name of research Group>.

For information about Research generally at UCLan please go to http://www.uclan.ac.uk/research/

All outputs in CLoK are protected by Intellectual Property Rights law, including Copyright law. Copyright, IPR and Moral Rights for the works on this site are retained by the individual authors and/or other copyright owners. Terms and conditions for use of this material are defined in the policies page. 


\title{
Low-energy electron diffraction study of potassium adsorbed on single-crystal graphite and highly oriented pyrolytic graphite
}

\author{
N. Ferralis, ${ }^{1}$ K. Pussi,${ }^{2}$ S. E. Finberg, ${ }^{3}$ J. Smerdon,${ }^{4}$ M. Lindroos,${ }^{2}$ R. McGrath,${ }^{4}$ and R. D. Dieh $1^{1}$ \\ ${ }^{1}$ Department of Physics and Materials Research Institute, Penn State University, University Park, Pennsylvania 16802, USA \\ ${ }^{2}$ Institute of Physics, Tampere University of Technology, P. O. Box 692, 33101 Tampere, Finland \\ ${ }^{3}$ Department of Natural Sciences, Bentley College, Waltham, Massachusetts 02452, USA \\ ${ }^{4}$ Department of Physics and Surface Science Research Centre, University of Liverpool, Liverpool, L69 3BX, United Kingdom
}

(Received 16 July 2004; published 6 December 2004)

\begin{abstract}
Potassium adsorption on graphite has been a model system for the understanding of the interaction of alkali metals with surfaces. The geometries of the $(2 \times 2)$ structure of potassium on both single-crystal graphite (SCG) and highly oriented pyrolytic graphite (HOPG) were investigated for various preparation conditions for graphite temperatures between 55 and $140 \mathrm{~K}$. In all cases, the geometry was found to consist of $\mathrm{K}$ atoms in the hollow sites on top of the surface. The K-graphite average perpendicular spacing is $2.79 \pm 0.03 \AA$, corresponding to an average $\mathrm{C}-\mathrm{K}$ distance of $3.13 \pm 0.03 \AA$, and the spacing between graphite planes is consistent with the bulk spacing of $3.35 \AA$. No evidence was observed for a sublayer of potassium. The results of dynamical LEED studies for the clean SCG and HOPG surfaces indicate that the surface structures of both are consistent with the truncated bulk structure of graphite.
\end{abstract}

DOI: $10.1103 /$ PhysRevB.70.245407

PACS number(s): 68.35.-p, 68.43.-h, 61.14.Hg

\section{INTRODUCTION}

Potassium adsorption on graphite continues to be studied as a model system because of the wide range of electronic states of $\mathrm{K}$ in this system, ${ }^{1-8}$ and of the intercalation process ${ }^{9}$ which is clearly present at temperatures above about $160 \mathrm{~K}$. Potassium dosed onto graphite at sufficiently low temperatures has been known to form a $(2 \times 2)$ superlattice structure since at least $1986 .{ }^{10}$ That study and earlier studies ${ }^{11-14}$ showed that when either single crystal graphite (SCG) or highly oriented pyrolytic graphite (HOPG) is exposed to a potassium flux, intercalation of the potassium occurs for sample temperatures higher than $200 \mathrm{~K}$, or when using a hot $\mathrm{K}$ source. Later, more detailed studies on HOPG at $90 \mathrm{~K}$ indicated little or no intercalation, and the evolution of the overlayer included a phase of widely dispersed $\mathrm{K}$ atoms at low coverages followed by a condensation into a $(2 \times 2)$ structure. ${ }^{15,16} \mathrm{~A}$ similar result was obtained for a He-atom scattering study of K dosed on SCG. ${ }^{17}$ The He-atom scattering study provided a clear indication that potassium forms an overlayer at $90 \mathrm{~K}$ from the intensity oscillations of the specular He reflection. Other He-atom scattering studies measured the phonon dispersion curves of the $\mathrm{K}$ overlayer and these agreed well with model calculations that assumed a $\mathrm{K}$ overlayer but no intercalate. ${ }^{18,19}$ Later studies to investigate the extent of intercalation under the typical low-pressure, lowtemperature conditions used in surface science experiments indicated that the stability of the $\mathrm{K}$ structure is dependent upon coverage. At the lowest coverages, intercalation can occur at temperatures as low as $50 \mathrm{~K}$, whereas multilayers are stable even at $150 \mathrm{~K} .{ }^{20}$ That is, the highly mobile dispersed phase can intercalate even at low temperatures, but once the solid layer is formed, intercalation is inhibited. The range of temperatures reported in various papers for the presence of appreciable intercalation likely can be attributed to differences in the defect density of the graphite and also the length of time required for the experiment.
Following this body of work, the interpretation of the $(2 \times 2)$ structure formed by dosing $\mathrm{K}$ at temperatures lower than about $100 \mathrm{~K}$ was widely assumed to be an overlayer structure. However, a recent photoemission study of $\mathrm{K}$ on HOPG suggested that there may be an underlying $(2 \times 2)$ layer of potassium beneath the top carbon layer which is more stable than a potassium overlayer, and that it may form before the overlayer. ${ }^{21}$ The evidence for this is the observation of two photoemission peaks. At $90 \mathrm{~K}$, the peak at the higher binding energy appears first and is attributed to an intercalated $(2 \times 2)$ layer of $\mathrm{K}$ because it remains stable upon further adsorption. The second peak appears later during the deposition and is attributed to a $(2 \times 2) \mathrm{K}$ overlayer. At lower temperatures, the size of the second (overlayer) peak is somewhat larger relative to the first peak, which is interpreted as being due to an inhibition of intercalation at low temperature. Furthermore, when the temperature is raised to $200 \mathrm{~K}$, the peak attributed to an intercalate remains stable, whereas the peak attributed to the overlayer disappears for temperatures above about $100 \mathrm{~K}$.

Because there has been so much study of the phases of $\mathrm{K}$ on graphite, and because the interpretation of such experiments depends on knowing if the $\mathrm{K}$ is adsorbed on or intercalated in the graphite, or both, we thought it very important to carry out a structural study of the $(2 \times 2)$ phase. In principle, it should not be difficult to distinguish the two types of structures since the intercalation of $\mathrm{K}$ will cause a significant expansion of the carbon layers. Structures interpreted as K intercalated under the first carbon layer of SCG have been studied before using low-energy electron diffraction (LEED), ${ }^{14}$ but $(2 \times 2)$ order was not observed in those studies. In this study, we carried out dynamical LEED studies of the $(2 \times 2)$ structures of $\mathrm{K}$ on both SCG and HOPG. The LEED studies on SCG provide higher-quality data, but since the intercalation process may depend on the type of graphite used, we also carried out experiments using HOPG. The ex- 


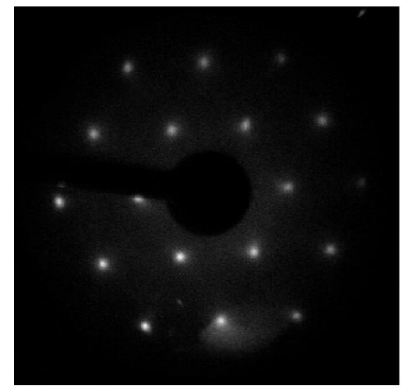

(a)

FIG. 1. LEED patterns for clean (a) SCG and (b) HOPG at an incident beam energy of $266 \mathrm{eV}$.

periments were carried out under various temperature and dosing conditions in order to attempt to form both $\mathrm{K}$ overlayers and underlayers.

\section{EXPERIMENT}

These experiments were performed with two different types of graphite substrates, single crystal graphite flakes (SCG) and HOPG (ZYA grade). In both cases, the samples were cleaved with Scotch tape just before inserting into the ultrahigh vacuum chamber (base pressure $3 \times 10^{-11} \mathrm{mbar}$ ), and they were heated for about a minute at $800-900{ }^{\circ} \mathrm{C}$ to remove residual impurities. The sample was attached to a liquid $\mathrm{He}$ cryostat by means of a copper braid, which allowed cooling to about $50 \mathrm{~K}$ in these experiments. The potassium was dosed onto the sample using a SAES Getters source that was mounted in a shuttered, collimated holder. The shutter allowed reproducible dosing.

The LEED experiments were performed on a commercial VG 3-grid optics, and the LEED patterns were acquired using a CCD camera interfaced to a PC. LEED patterns from the clean SCG and HOPG surfaces are shown in Fig. 1. The LEED pattern from HOPG consists of rings instead of spots because HOPG comprises a large number of micron-size crystallites having random in-plane orientations, and the diffraction pattern is an incoherent superposition of many single-crystal diffraction patterns. For single-crystal patterns, the intensities of the beams were extracted by integrating the intensity over a circle around each diffraction spot. Background intensity equal to the average intensity of the perimeter of the integration circle was then subtracted from the integrated intensity. The extracted intensities of equivalent beams were then averaged. For the HOPG patterns, the average ring intensities were extracted by using a set of 30-50 integration windows arranged on the circumference of the rings. Because of the proximity of the rings to each other, background measurements were not generally feasible, and instead, a smoothly varying background was subtracted from the resulting intensity curves.

The LEED $I(E)$ calculations used the Barbieri/Van Hove Tensor LEED package. Phase shifts up to desired value of $l_{\max }$ were used to describe the scattering properties of carbon and potassium. The phase shifts were calculated using the Barbieri-Van Hove ${ }^{22}$ phase shift package. Theoryexperiment agreement was tested using the Pendry $r$ factor
TABLE I. Experimental and best-fit structural parameters for clean graphite. The energy range corresponds to the length of the experimental datasets $z_{i j}$ (average) is the interlayer spacing between the centers of mass of the layers $i$ and $j$ and "rumple" corresponds to height difference between the two inequivalent atoms in the layer. $\mathrm{V}_{i}$ is the imaginary part of the inner potential, $l_{\max }$ is the maximum angular momentum quantum number used for the calculation of the potential, and $\Theta_{D}$ is the fitted Debye temperature. $R_{P}$ is the Pendry $r$ factor. The bulk interlayer spacing for graphite is $3.35 \AA$.

\begin{tabular}{lll} 
Parameter & $\mathrm{SCG}(0001)($ Ref. 24) & $\mathrm{HOPG}(0001)$ \\
Energy range & $1015 \mathrm{eV}$ & $900 \mathrm{eV}$ \\
Sample temperature & $131 \mathrm{~K}$ & $73 \mathrm{~K}$ \\
$z_{12}$ (average) & $3.37 \pm 0.04 \AA$ & $3.35 \pm 0.05 \AA$ \\
$z_{23}$ (average) & $3.40 \pm 0.06 \AA$ & $3.36 \pm 0.10 \AA$ \\
$z_{34}$ (average) & $3.32 \pm 0.08 \AA$ & $3.31 \pm 0.13 \AA$ \\
Rumple (layer 1) & $0.03 \pm 0.05 \AA$ & $0.04 \pm 0.04 \AA$ \\
Rumple (layer 2) & $0.02 \pm 0.04 \AA$ & $0.00 \pm 0.07 \AA$ \\
Rumple (layer 3) & $0.03 \pm 0.08 \AA$ & $0.00 \pm 0.13 \AA$ \\
$V_{i}$ & $-4 \mathrm{eV}$ & $-5 \mathrm{eV}$ \\
$I_{\text {max }}$ & 10 & 8 \\
$\Theta_{D}$ & $800 \mathrm{~K}$ & $420 \mathrm{~K}$ \\
$R_{P}$ & 0.25 & 0.28 \\
\hline \hline
\end{tabular}

and error bars were calculated using the Pendry RR function. ${ }^{23}$ The structural parameters that were optimized were the interlayer spacing of the first three layers of graphite, the interlayer rumpling between the two different types of $\mathrm{C}$ atoms in the graphite unit cell (first three layers), and the K-graphite site and distance. For the SCG studies, the relative amount of the two graphite terminations was an adjustable parameter, ${ }^{24}$ and for HOPG it was assumed to be a 50:50 distribution of terminations. The nonstructural parameters that were optimized were the layer-dependent Debye temperature of the graphite and of the $\mathrm{K}$, and the real and imaginary parts of the inner potential.

\section{RESULTS}

\section{A. Clean HOPG}

Although the surface structure of HOPG was not expected to be different from SCG, the LEED analysis was carried out as a comparison of the measurement technique, since the procedures for extracting the intensities are different for the two substrates. The dynamical LEED analysis for SCG has been presented before ${ }^{24}$ and it was found that the SCG surface is essentially an unrelaxed truncation of the bulk structure. Table I presents the results of that study along with the results of the HOPG study presented here. Figure 2 shows the LEED spectra from the clean HOPG. These spectra differ visually from those from SCG (Ref. 24) in that the peaks are broader and lack some of the fine structure. This is most likely due to the averaging over crystallites having different (0001) orientations. The width of the distribution of (0001) orientations is about $0.4^{\circ}$ for this grade of HOPG. Although 


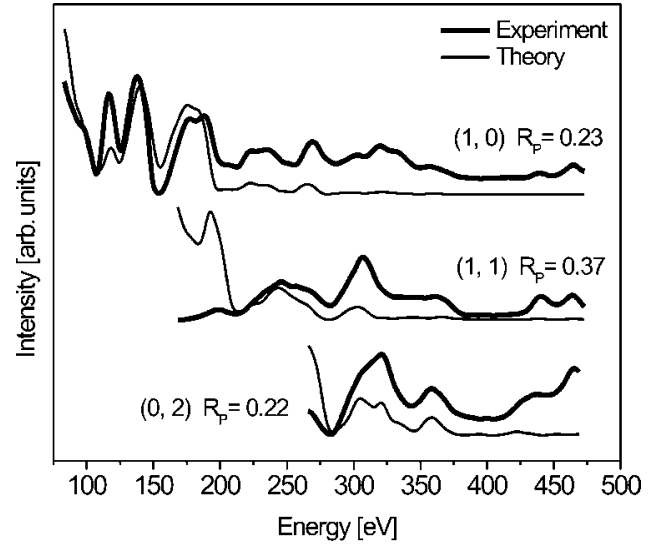

FIG. 2. Experimental and calculated spectra for clean HOPG $(0001)$ at $73 \mathrm{~K}$. The indices correspond to diffraction spots that are equivalent to the three rings [shown in Fig. 1(b)], which correspond to rings 3,6 , and 8 in Fig. 6 .

the spectra from SCG and HOPG differ in the detail of their features, the locations of the major peaks are the same, and as seen from Table I, the results of the LEED analysis are similar for the two types of graphite. For both, the interlayer relaxation and intralayer rumpling are bulklike, within the precision of this determination. Therefore, apart from crystallite size and orientation, the clean HOPG surface is essentially the same as the single crystal surface. The effect of the polycrystallinity of the HOPG in terms of the LEED experiment is to reduce the precision of the structural parameters, but the level of correspondence between the two types of graphite is good.

\section{B. Adsorption of potassium on SCG and HOPG}

The experimental procedures for forming the $(2 \times 2)$ structure of potassium on both SCG and HOPG were adapted from those used in the earlier photoemission study of $\mathrm{K}$ on
HOPG. ${ }^{21}$ Structures having $(2 \times 2)$ symmetry were formed following procedures that varied according to the dosing time, dosing temperature and post-dose annealing temperature. Dosing temperatures were varied between 55 and $140 \mathrm{~K}$ and post-dose anneals were done at temperatures up to 140 $\mathrm{K}$. After preparation of the film, the LEED patterns were recorded and the intensity curves extracted. In light of the results reported in the earlier photoemission study, a particular emphasis was placed on conditions that were reported favorable for the formation of a $\mathrm{K}$ underlayer, in particular those formed at higher temperatures and at relatively low coverage. There were no qualitative differences between the intensity spectra generated by the different preparation methods. The spectra from the SCG structure include more fine structure, as for the clean surface, but the locations of the diffraction peaks were the same for all $(2 \times 2)$ structures formed. The sections below provide details of the LEED analyses.

\section{C. $(2 \times 2)$ structure analyses}

The LEED spectra for the $(2 \times 2)$ structure formed by $\mathrm{K}$ on SCG at $65 \mathrm{~K}$ are shown in Fig. 3. The analysis of the data was started by calculating the spectra for the $\mathrm{K}$ atoms in different sites above the bulk-truncated graphite surface. Four different high-symmetry adsorption sites were explored: top A, top B, hollow and bridge, as labeled in Fig. 4. The two top sites differ by the fact that top A has no carbon atom in the second layer beneath the $\mathrm{K}$ atom, whereas top $\mathrm{B}$ does have a carbon atom in that location. On each site, the potassium atom was moved vertically in the range $r(\mathrm{C})$ $+r(\mathrm{~K}) \pm 30 \%$, where $r(\mathrm{C})$ is the van der Waals radius of the carbon $(1.70 \AA)$ and $r(\mathrm{~K})$ is the bulk radius of the potassium $(2.27 \AA)$. The $r$ factors after this preliminary search were $0.68,0.66,0.52$, and 0.63 for top $\mathrm{A}$, top $\mathrm{B}$, hollow, and bridge, respectively, favoring the hollow site.

Structures with K under the top graphite layer were also investigated. Structures, including relaxations, with an un-

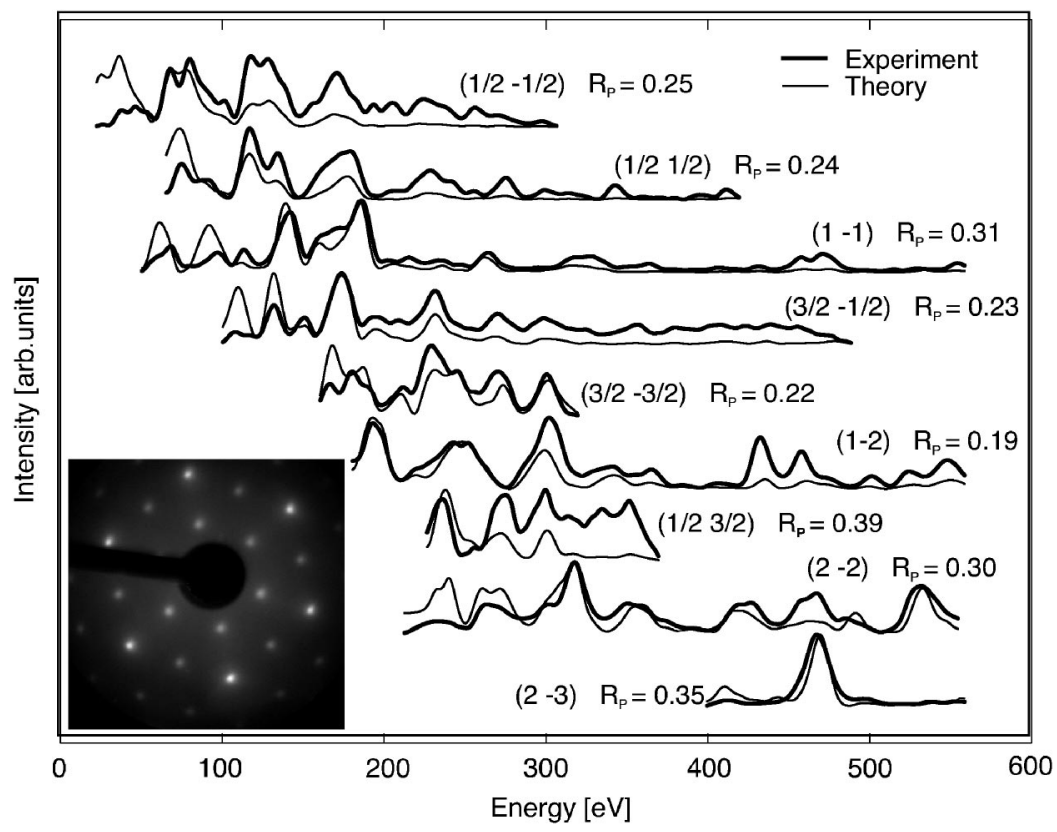

FIG. 3. Experimental and calculated spectra for SCG(0001)- $(2 \times 2)-\mathrm{K}$ at $65 \mathrm{~K}$. Inset: LEED pattern at $135 \mathrm{eV}$. The bright spots in the outer ring are integer-order beams and the others are $(2 \times 2)$ superlattice beams. 


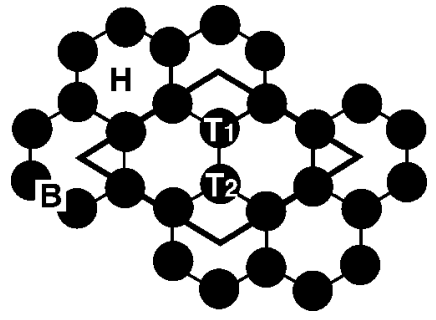

FIG. 4. Diagram of adsorption sites for the basal plane of graphite. $H, T$, and $B$ denote hollow, top, and bridge sites, respectively. $T 1$ and $T 2$ differ in that $T 1$ has a carbon atom beneath it in the second graphene layer, whereas $T 2$ does not.

derlayer of $\mathrm{K}$ atoms in the hollow positions having stacking sequences $A-\mathrm{K}-A B A B$ and $A-\mathrm{K}-B A B A$ produced the $r$ factors 0.80 and 0.82 , respectively. Structures with both an underlayer and an overlayer of $\mathrm{K}$ atoms (in different hollow sites) having a stacking sequence $\mathrm{K}-A-\mathrm{K}-A B A B$ produced a best $r$ factor of 0.69 . The reduction in $r$ factor that occurs when adding the $\mathrm{K}$ overlayer to the underlayer structure is indicative of the presence of a $(2 \times 2) \mathrm{K}$ overlayer, but even with relaxations, the underlayer structures did not produce the level of agreement of just the overlayer in the hollow sites. Upon refinement of the structural and nonstructural parameters, the hollow-site overlayer produced an $r$ factor of 0.27 , which is only slightly higher than that obtained for the clean SCG. The best-fit parameters are given in Table II.

The LEED spectra for the $(2 \times 2)$ structure formed by $\mathrm{K}$ on HOPG at $62 \mathrm{~K}$ are shown in Fig. 5. A visual comparison of these spectra with those in Fig. 3 shows the same broadening of the features that occurs in the case of the clean surface. The extraction of intensities in this case was a more difficult than for clean HOPG due to the closer proximity of the rings. This can be seen easily in the diffraction pattern diagram shown in Fig. 6, which shows the first eight diffraction rings observed for the $(2 \times 2)$ superlattice. On clean HOPG, only those labeled 3, 6, and 8 are present, and their
TABLE II. Experimental and best-fit structural parameters for the potassium $(2 \times 2)$ structure on two types of graphite. The energy range corresponds to the length of the experimental datasets. $\mathrm{r}_{\mathrm{K}-\mathrm{C}}$ is the $\mathrm{K}-\mathrm{C}$ bond length, $z_{i j}$ (average) is the interlayer spacing between the centers of mass of the layers $i$ and $j$, and "rumple" corresponds to height difference between the two nonequivalent atoms in the layer. $V_{i}$ is the imaginary part of the inner potential and was taken to vary as $E^{1 / 3}$, normalized to $-4 \mathrm{eV}$ at $120 \mathrm{eV}$ and $90 \mathrm{eV}$ for SCE and HOPG, respectively. $l_{\max }$ is the maximum angular momentum quantum number used for the calculation of the potential. $R_{P}$ is the Pendry $r$ factor. The bulk interlayer spacing for graphite is $3.35 \AA$.

\begin{tabular}{lll}
\hline \hline Parameter & $\mathrm{SCG}(0001)$ & $\mathrm{HOPG}(0001)$ \\
Energy range & $2800 \mathrm{eV}$ & $1900 \mathrm{eV}$ \\
Sample temperature & $65 \mathrm{~K}$ & $62 \mathrm{~K}$ \\
$r_{\mathrm{K}-\mathrm{C}}$ (average) & $3.13 \pm 0.03 \AA$ & $3.08 \pm 0.07 \AA$ \\
$z_{\mathrm{K}-\mathrm{C}}$ (average) & $2.79 \pm 0.03 \AA$ & $2.70 \pm 0.06 \AA$ \\
$z_{12}$ & $3.34 \pm 0.04 \AA$ & $3.34 \pm 0.05 \AA$ \\
$z_{23}$ & $3.37 \pm 0.04 \AA$ & $3.37 \pm 0.12 \AA$ \\
$Z_{34}$ & $3.37 \pm 0.07 \AA$ & $3.39 \pm 0.14 \AA$ \\
Rumple (layer 1) & $0.01 \pm 0.03 \AA$ & $0.04 \pm 0.05 \AA$ \\
Rumple (layer 2) & $0.00 \pm 0.05 \AA$ & $0.02 \pm 0.09 \AA$ \\
Rumple (layer 3) & $0.01 \pm 0.07 \AA$ & $0.02 \pm 0.14 \AA$ \\
$V_{i}$ & $\mathrm{E}^{1 / 3}$ & $\mathrm{E}^{1 / 3}$ \\
$I_{\text {max }}$ & 12 & 12 \\
$R_{P}$ & 0.27 & 0.40 \\
\hline \hline
\end{tabular}

wider spacing allows an unambiguous extraction of the intensities. For the $(2 \times 2)$ structure, however, some of the rings are so close that their intensities can overlap. Therefore not all rings could be measured, and in some cases, notably for rings 6 and 8 , it is possible that some contribution of the adjacent rings is present. Nevertheless, for the measured rings, there is a good correspondence between the intensities measured on HOPG with those measured on SCG. The best-

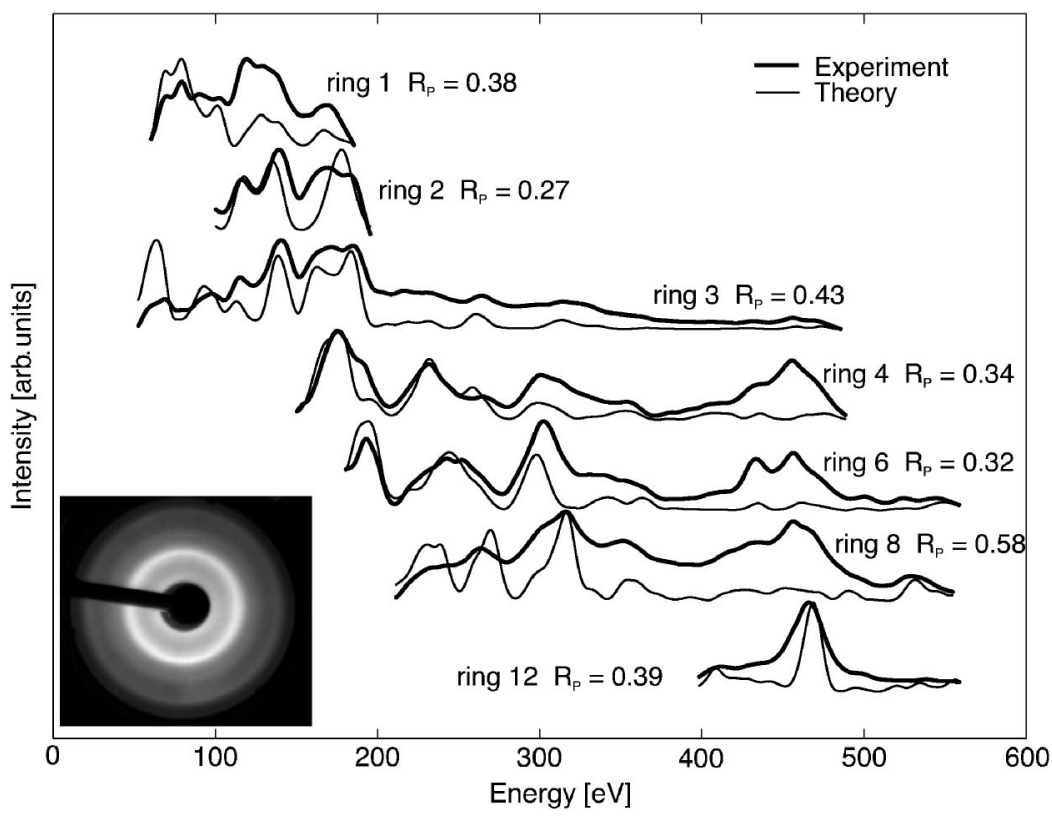

FIG. 5. Experimental and calculated spectra for $\mathrm{HOPG}(0001)-(2 \times 2)-\mathrm{K}$ at $62 \mathrm{~K}$. The ring labels correspond to Fig. 6. Inset: LEED pattern at $179 \mathrm{eV}$. The visible rings correspond to 1,2 , and 3 in Fig. 6. 


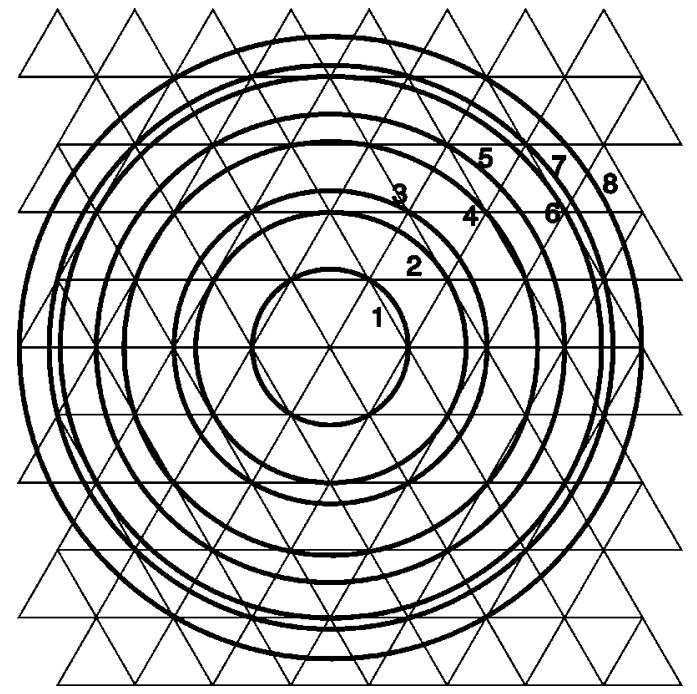

FIG. 6. Schematic diagram of the diffraction patterns from graphite $(0001)-(2 \times 2)$ for a SCG and HOPG. The $(2 \times 2)$ superlattice beams for SCG will occur at all of the intersections on the triangular grid. The intensity rings observed for HOPG are effectively those points dispersed azimuthally. The ring numbers correspond to those used in Fig. 5.

fit parameters are given in Table II. While the $62 \mathrm{~K}$ data are presented here, a comparison of the LEED spectra obtained under different adsorption conditions (varying $\mathrm{K}$ dose and temperature) shows that under all conditions studied, the diffraction curves are qualitatively identical, i.e., the peaks occur at the same energies.

\section{DISCUSSION}

The results presented here indicate that the surface structure of clean HOPG is the same as that of SCG. They also indicate that the method used to analyze the intensities from the HOPG produces results consistent with those of SCG. The misorientation of the crystallites in the HOPG results in somewhat larger uncertainties in the structural parameters. The nonstructural parameters such as the fitted Debye temperature and the imaginary part of the inner potential also indicated greater disorder in the HOPG.

The structures determined for the potassium $(2 \times 2)$ structures on SCG and HOPG are essentially the same: both are consistent with a structure which $\mathrm{K}$ occupies overlayer hollow sites, but not intercalate sites. The lack of intercalation is clearly evident in the LEED spectra from the integer order beams, because the intercalate would create a large expansion of the carbon layers, causing a significant shift in the peaks of the integer order LEED beams. A comparison of the first-order graphite spot/ring for the clean surfaces and for different $\mathrm{K}$ dosing conditions is shown in Fig. 7. While the

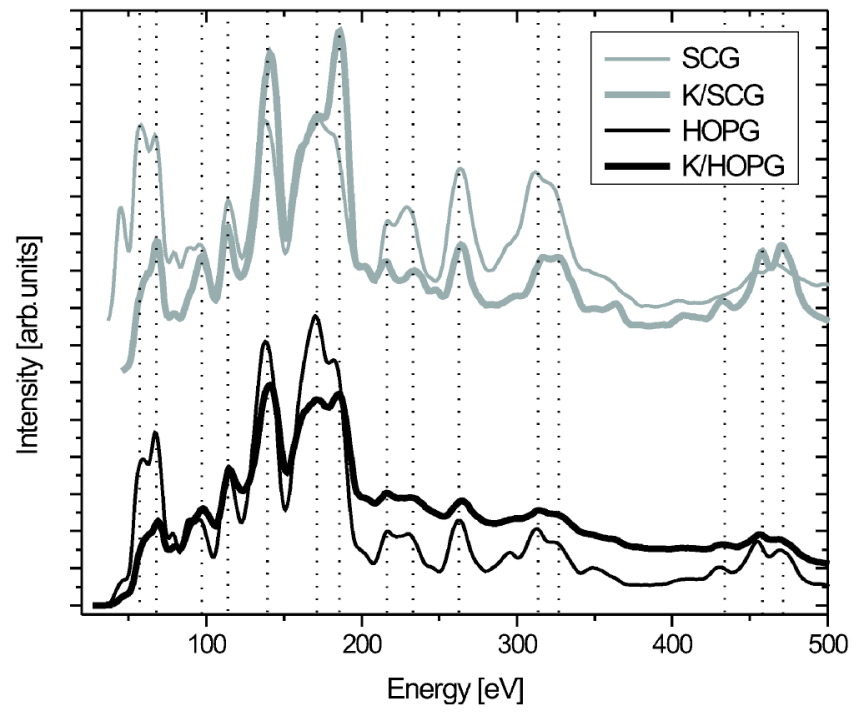

FIG. 7. Intensity of the lowest-integer-order beam for graphite, corresponding to ring 3 in Fig. 6, for SCG and HOPG, and for the $(2 \times 2)$ structure on both substrates.

relative intensities change, the locations of the peaks do not shift in energy when $\mathrm{K}$ is adsorbed indicating that the graphite structure does not change, and therefore there is no appreciable intercalation in the top layer.

This result does little to explain the origin of the two peaks observed in the earlier photoemission study, ${ }^{21}$ but it does indicate that neither of them is due to an intercalated $(2 \times 2)$ structure of potassium. ${ }^{25}$ Since one of them is stable to relatively high temperatures, it suggests that this might be due to $\mathrm{K}$ adsorbed at some defect sites such as crystallite edges and steps, which are numerous in HOPG. The second peak, however, does appear to be consistent with a potassium overlayer structure since its thermal stability is similar to that observed in other studies, and potassium does not appear to form a $(2 \times 2)$ underlayer structure under these conditions.

To summarize, we have shown that potassium dosed onto either SCG or HOPG between 50 and $150 \mathrm{~K}$ forms a structure having $(2 \times 2)$ symmetry, and that this structure consists of a potassium overlayer on the graphite surface. The graphite structure is consistent with the bulk structure of graphite, and the $\mathrm{K}$ atoms reside at the centers of the hollows on the graphite. The average perpendicular $\mathrm{K}$-graphite distance is $2.79 \pm 0.03 \AA$ for the SCG structure, which is consistent with the $2.70 \pm 0.06 \AA$ value obtained for the HOPG structure.

\section{ACKNOWLEDGMENTS}

We gratefully acknowledge financial support from the National Science Foundation (Grant Nos. DMR-0208520, DGE-9979579, and DGE-0338240), the UK EPSRC, and the Finnish National Graduate School in Materials Physics. 
${ }^{1}$ B. Hellsing, D. V. Chakarov, L. Osterlund, V. P. Zhdanov, and B. Kasemo, J. Chem. Phys. 106, 982 (1997).

${ }^{2}$ D. V. Chakarov, P. Sjovall, and B. Kasemo, J. Phys.: Condens. Matter 5, 2903 (1993).

${ }^{3}$ D. V. Chakarov, L. Osterlund, B. Hellsing, V. P. Zhdanov, and B. Kasemo, Surf. Sci. 311, L724 (1994).

${ }^{4}$ D. V. Chakarov, L. Osterlund, B. Hellsing, and B. Kasemo, Appl. Surf. Sci. 106, 186 (1996).

${ }^{5}$ B. Hellsing, D. V. Chakarov, L. Osterlund, V. P. Zhdanov, and B. Kasemo, Surf. Sci. 363, 247 (1996).

${ }^{6}$ P. Bennich, C. Puglia, P. A. Bruhwiler, A. Nilsson, A. J. Maxwell, A. Sandell, N. Martensson, and P. Rudolf, Phys. Rev. B 59, 8292 (1999).

${ }^{7}$ L. Osterlund, D. V. Chakarov, and B. Kasemo, Surf. Sci. 420, 174 (1999).

${ }^{8}$ M. Gleeson, B. Kasemo, and D. Chakarov, Surf. Sci. 524, L77 (2003).

${ }^{9}$ P. Sjovall, Surf. Sci. 345, L39 (1996).

${ }^{10}$ M. T. Johnson, H. I. Starnberg, and H. P. Hughes, Surf. Sci. 178, 290 (1986).

${ }^{11}$ N. J. Wu and A. Ignatiev, J. Vac. Sci. Technol. 20, 896 (1982).

${ }^{12}$ N. J. Wu and A. Ignatiev, J. Vac. Sci. Technol. 20, 896 (1982).

${ }^{13}$ N. J. Wu and A. Ignatiev, Solid State Commun. 46, 59 (1983).
${ }^{14}$ N. J. Wu and A. Ignatiev, Phys. Rev. B 28, 7288 (1983).

${ }^{15}$ Z. Y. Li, K. M. Hock, and R. E. Palmer, Phys. Rev. Lett. 67, 1562 (1991).

${ }^{16}$ Z. Y. Li, K. M. Hock, R. E. Palmer, and J. F. Annett, J. Phys.: Condens. Matter 3, S103 (1991).

${ }^{17}$ J. Cui, J. D. White, R. D. Diehl, J. F. Annett, and M. W. Cole, Surf. Sci. 279, 149 (1992).

${ }^{18}$ J. D. White, J. Cui, M. Strauss, R. D. Diehl, F. Ancilotto, and F. Toigo, Surf. Sci. 307-309, 1134 (1994).

${ }^{19}$ J. Cui, J. D. White, and R. D. Diehl, Solid State Commun. 293, L841 (1993).

${ }^{20}$ J. C. Barnard, K. M. Hock, and R. E. Palmer, Surf. Sci. 287/288, 178 (1993).

${ }^{21}$ M. Breitholtz, T. Kihlgren, S.-Å. Lindgren, and L. Wallden, Phys. Rev. B 66, 153401 (2002).

${ }^{22}$ A. Barbieri and M. A. Van Hove (private communication).

${ }^{23}$ J. B. Pendry, J. Phys. C 13, 937 (1980).

${ }^{24}$ K. Pussi, J. Smerdon, N. Ferralis, M. Lindroos, R. McGrath, and R. D. Diehl, Surf. Sci. 548, 157 (2004).

${ }^{25}$ Since acceptance of this paper, we have learned that the peak originally attributed to an underlayer of $\mathrm{K}$ has now been identified as diffraction-related feature of the overlayer. L. Wallden, private communication. 